\title{
Normal Approximations of the Arithmetic of Mixed Fuzzy Numbers
}

\section{Yi-Fang Chen and Hui-Chin Tang}

Department of Industrial Engineering and Management, National Kaohsiung University of Applied Sciences, Kaohsiung 80778, Taiwan, R.O.C.

\begin{abstract}
In fuzzy group decision making problems and fuzzy shortest path problems, the addition and subtraction are the basic problems. For the mixed normal fuzzy numbers and trapezoidal fuzzy numbers, the addition and subtraction operations are approximated by the normal fuzzy numbers in this paper. The behaviors of approximated normal fuzzy numbers are the same as those of the normal distributions from the viewpoint of probability. An application of the addition and subtraction operations of mixed fuzzy numbers to the fuzzy sample mean is also proposed.
\end{abstract}

Keywords: fuzzy number; normal fuzzy number; addition and subtraction; approximation. 


\section{Introduction}

For solving multiple judges, multiple criteria decision making problems in a fuzzy environment, aggregating fuzzy performance ratings and fuzzy weights through all judges and aggregating fuzzy ratings with fuzzy weight are two basic steps. Fuzzy addition and fuzzy multiplication are two operations to aggregate different judges and criteria. Solving shortest path problems on a network with mixed fuzzy arc lengths is to determine all nondominated paths based on the order relation among fuzzy numbers. Calculating the fuzzy distance along the path is the sum of associated arc lengths. Therefore, the fuzzy addition and subtraction operations are the fundamental problems in the group decision making problems and the fuzzy shortest path problems. For more information on the fuzzy group decision making problems and fuzzy shortest path problems, we refer the reader to references [1-4] and [5-7], respectively.

In fuzzy arithmetic, addition and subtraction operations are two fundamental problems of fuzzy optimization and decision making. For the same type of fuzzy numbers, the results of addition and subtraction operations are the same ones. For the mixed fuzzy numbers, four types of approximations are proposed in the literature: defuzzification, interval approximation, triangular fuzzy number and trapezoidal approximation [8-11]. From the viewpoint of probability, normal fuzzy number 
approximation is the alternative one. This paper adopts the normal fuzzy number approximation to analyze the behaviors of the addition and subtraction operations for the mixed fuzzy numbers.

The organization of this paper is as follows. Section 2 briefly reviews the fuzzy sets. Section 3 presents the addition and subtraction of the same and mixed fuzzy numbers. An application of the addition and subtraction operations of the same and mixed fuzzy numbers to the fuzzy sample mean is presented in section 4. Finally, we end with some concluding remarks.

\section{Fuzzy Sets}

We firstly review the basic notations of fuzzy sets. Consider a fuzzy set $A$ defined on a universal set of real numbers $\mathcal{R}$ by the membership function $A(\mathrm{x})$, where $A(x): \Re \rightarrow[0,1]$.

Definition 1. Let $A$ be a fuzzy set. The support of $A$ is the crisp set $S_{A}=\{x \in \Re \mid A(x)>0\} . A$ is called normal when $\sup _{x \in S_{A}} A(x)=1$. An $\alpha-$ cut of $A$ is a crisp set $A_{\alpha}=\{x \in \mathfrak{R} \mid A(x) \geq \alpha\} . A$ is convex if, and only if, each of its $\alpha$-cut is a convex set.

Definition 2. A normal and convex fuzzy set whose membership function is piecewise continuous is called a fuzzy number. 
Definition 3. A trapezoidal fuzzy number $A$, denoted $A(a, b, c, d)$, is a fuzzy number with membership function given by

$$
A(\mathrm{x})=\left\{\begin{array}{cc}
\frac{x-a}{b-a} & \text { if } a \leq x \leq b \\
\frac{d-x}{d-c} & \text { if } c \leq x \leq d \\
0 & \text { otherwise }
\end{array}\right.
$$

where $-\infty<a \leq b \leq c \leq d<\infty$. An $\alpha-$ cut of $A$ is

$$
A_{\alpha}=[L, U]=[a+(b-a) \alpha, d-(d-c) \alpha]
$$

The set of all trapezoidal fuzzy numbers on $\mathcal{R}$ is denoted by $\operatorname{TF}(\mathcal{R})$.

Definition 4. A normal fuzzy number $A$, denoted $A(m, \sigma)$, is a fuzzy number with membership function given by

$$
A(\mathrm{x})=e^{-\left(\frac{x-m}{\sigma}\right)^{2}}
$$

where $m \in \mathcal{R}$ and $\sigma>0$. An $\alpha-$ cut of $A$ is

$$
A_{\alpha}=[L, U]=[m-\sigma \sqrt{-\ln \alpha}, m+\sigma \sqrt{-\ln \alpha}]
$$

The set of all normal fuzzy numbers on $\mathcal{R}$ is denoted by $N(\mathcal{R})$.

Definition 5. Let $A$ and $B$ be two fuzzy numbers and $\times$ be an operation on $\mathcal{R}$, such as $+,-, *, \div \ldots$ By extension principle, the extended operation $\otimes$ on fuzzy numbers can be defined by

$$
\mu_{A \otimes B}(z)=\sup _{x, y: z=x \times y} \min \{A(x), B(y)\} .
$$




\section{Arithmetic of the Same and Mixed Fuzzy Numbers}

This section analyzes the addition and subtraction of the same and mixed fuzzy numbers. For the same fuzzy numbers, we firstly consider two trapezoidal fuzzy numbers $A\left(a_{1}, b_{1}, c_{1}, d_{1}\right)$ and $B\left(a_{2}, b_{2}, c_{2}, d_{2}\right)$. It follows that

$$
\begin{gathered}
A_{\alpha}=\left[L_{1}, U_{1}\right]=\left[a_{1}+\left(b_{1}-a_{1}\right) \alpha, d_{1}-\left(d_{1}-c_{1}\right) \alpha\right] \\
B_{\alpha}=\left[L_{2}, U_{2}\right]=\left[a_{2}+\left(b_{2}-a_{2}\right) \alpha, d_{2}-\left(d_{2}-c_{2}\right) \alpha\right] .
\end{gathered}
$$

Let $\gamma, \beta \geq 0$, the addition operation $\gamma A+\beta B$ is

$$
\begin{gathered}
\gamma \mathrm{A}_{\alpha}+\beta \mathrm{B}_{\alpha}=\gamma\left[L_{1}, U_{1}\right]+\beta\left[L_{2}, U_{2}\right] \\
=\left[\gamma a_{1}+\beta a_{2}+\left(\gamma\left(b_{1}-a_{1}\right)+\beta\left(b_{2}-a_{2}\right)\right) \alpha, \gamma d_{1}+\beta d_{2}-\left(\gamma\left(d_{1}-c_{1}\right)+\right.\right. \\
\left.\left.\beta\left(d_{2}-c_{2}\right)\right) \alpha\right]
\end{gathered}
$$

so

$$
\gamma A+\beta B=\left(\gamma a_{1}+\beta a_{2}, \gamma b_{1}+\beta b_{2}, \gamma c_{1}+\beta c_{2}, \gamma d_{1}+\beta d_{2}\right)
$$

Similarly the subtraction operation is

$$
\gamma A-\beta B=\left(\gamma a_{1}-\beta d_{2}, \gamma b_{1}-\beta c_{2}, \gamma c_{1}-\beta b_{2}, \gamma d_{1}-\beta a_{2}\right)
$$

For the two normal fuzzy numbers $A\left(m_{1}, \sigma_{1}\right)$ and $B\left(m_{2}, \sigma_{2}\right)$, we have that

$$
\begin{gathered}
A_{\alpha}=\left[m_{1}-\sigma_{1} \sqrt{-\ln \alpha}, m_{1}+\sigma_{1} \sqrt{-\ln \alpha}\right] \\
B_{\alpha}=\left[m_{2}-\sigma_{2} \sqrt{-\ln \alpha}, m_{2}+\sigma_{2} \sqrt{-\ln \alpha}\right] .
\end{gathered}
$$

Let $\gamma, \beta \geq 0$, the addition operation and subtraction operation are 


$$
\gamma A+\beta B=\left(\gamma m_{1}+\beta m_{2}, \gamma \sigma_{1}+\beta \sigma_{2}\right)
$$

and

$$
\gamma A-\beta B=\left(\gamma m_{1}-\beta m_{2}, \gamma \sigma_{1}+\beta \sigma_{2}\right)
$$

respectively.

From the viewpoint of probability, these results coincide with the mean and standard deviation of the normal distribution.

Theorem 1. (1) Let $A\left(a_{1}, b_{1}, c_{1}, d_{1}\right)$ and $B\left(a_{2}, b_{2}, c_{2}, d_{2}\right)$ be two trapezoidal fuzzy numbers and $\gamma, \beta \geq 0$. Then we have that

$$
\gamma A+\beta B=\left(\gamma a_{1}+\beta a_{2}, \gamma b_{1}+\beta b_{2}, \gamma c_{1}+\beta c_{2}, \gamma d_{1}+\beta d_{2}\right)
$$

and

$$
\gamma A-\beta B=\left(\gamma a_{1}-\beta d_{2}, \gamma b_{1}-\beta c_{2}, \gamma c_{1}-\beta b_{2}, \gamma d_{1}-\beta a_{2}\right)
$$

(2) Let $A\left(m_{1}, \sigma_{1}\right)$ and $B\left(m_{2}, \sigma_{2}\right)$ be two normal fuzzy numbers and $\gamma, \beta \geq 0$. Then we have that

$$
\gamma A+\beta B=\left(\gamma m_{1}+\beta m_{2}, \gamma \sigma_{1}+\beta \sigma_{2}\right)
$$

and

$$
\gamma A-\beta B=\left(\gamma m_{1}-\beta m_{2}, \gamma \sigma_{1}+\beta \sigma_{2}\right)
$$

For the mixed fuzzy numbers, we firstly consider the trapezoidal fuzzy number $A(a, b, c, d)$ and the normal fuzzy number $B(m, \sigma)$. It follows that 


$$
\begin{gathered}
A_{\alpha}=\left[L_{1}, U_{1}\right]=[a+(b-a) \alpha, d-(d-c) \alpha] \\
B_{\alpha}=\left[L_{2}, U_{2}\right]=[m-\sigma \sqrt{-\ln \alpha}, m+\sigma \sqrt{-\ln \alpha}]
\end{gathered}
$$

Let $\gamma, \beta \geq 0$, the addition operation is

$$
\begin{gathered}
C_{\alpha}=\left[L_{\mathrm{C}}, U_{\mathrm{C}}\right]=\gamma A_{\alpha}+\beta B_{\alpha}=\left[\gamma L_{1}+\beta L_{2}, \gamma U_{1}+\beta U_{2}\right] \\
=[\beta m+\gamma \mathrm{a}+\gamma(\mathrm{b}-\mathrm{a}) \alpha-\beta \sigma \sqrt{-\ln \alpha}, \beta m+\gamma \mathrm{d}-\gamma(\mathrm{d}-\mathrm{c}) \alpha+\beta \sigma \sqrt{-\ln \alpha}] .
\end{gathered}
$$

By the general regression model, we use the normal fuzzy number $C\left(m_{C}, \sigma_{C}\right)$ to approximate the fuzzy number $\gamma A+\beta B$. The least square approximation is stated formally below.

Theorem 2. Consider the regression model

$$
x_{i}=m+D \sigma \sqrt{-\ln \mathrm{y}_{i}}
$$

where $x_{i}=A+B \frac{i}{n}+C \sqrt{-\ln \frac{i}{n}}, \quad \mathrm{y}_{i}=i / n, \quad i=1,2, \ldots, n, \quad A, B, C \in \mathcal{R}$ and $D \in$ $\{-1,1\}$. Then the least squares estimators are

$$
\begin{gathered}
\widehat{m}=A+B \frac{8+8 n+\sqrt{2} \pi n}{4 n(4-\pi)} \\
\hat{\sigma}=D C+D B \frac{(-2+(-2+\sqrt{2}) n) \sqrt{\pi}}{2 n(4-\pi)} .
\end{gathered}
$$

As $n \rightarrow \infty$, it follows that

$$
\begin{gathered}
\widehat{m}=A+B \frac{8+\sqrt{2} \pi}{4(4-\pi)}=A+1.0359642 B \\
\hat{\sigma}=D C+D B \frac{(-2+\sqrt{2}) \sqrt{\pi}}{2(4-\pi)}=D C-0.604771 D B .
\end{gathered}
$$


Proof. We decompose $(0,1]$ into $n$ subintervals by inserting $\frac{1}{n}, \frac{2}{n}, \ldots, 1$ points of subdivision. Define

$$
\mathbb{X}=\left[\begin{array}{c}
x_{1} \\
x_{2} \\
\vdots \\
x_{n}
\end{array}\right]=\left[\begin{array}{c}
A+B \frac{1}{n}+C \sqrt{-\ln \frac{1}{n}} \\
A+B \frac{2}{n}+C \sqrt{-\ln \frac{2}{n}} \\
\vdots \\
A+B \frac{n}{n}+C \sqrt{-\ln \frac{n}{n}}
\end{array}\right]
$$

and

$$
\mathbb{Y}=\left[\begin{array}{cc}
1 & D \sqrt{-\ln \mathrm{y}_{1}} \\
1 & D \sqrt{-\ln \mathrm{y}_{2}} \\
\vdots & \vdots \\
1 & D \sqrt{-\ln \mathrm{y}_{n}}
\end{array}\right]=\left[\begin{array}{cc}
1 & D \sqrt{-\ln 1 / n} \\
1 & D \sqrt{-\ln 2 / n} \\
\vdots & \vdots \\
1 & D \sqrt{-\ln n / n}
\end{array}\right]
$$

The least squares estimators are

$$
\begin{gathered}
{\left[\begin{array}{c}
\hat{m} \\
\hat{\sigma}
\end{array}\right]=\left(\mathbb{Y}^{\prime} \mathbb{Y}\right)^{-1} \mathbb{Y}^{\prime} \mathbb{X}=} \\
\frac{1}{n \sum_{i=1}^{n}\left(-\ln \mathrm{y}_{i}\right)-\left(\sum_{i=1}^{n} \sqrt{-\ln \mathrm{y}_{i}}\right)^{2}}\left[\begin{array}{cc}
\sum_{i=1}^{n}\left(-\ln \mathrm{y}_{i}\right) & -D \sum_{i=1}^{n} \sqrt{-\ln \mathrm{y}_{i}} \\
-D \sum_{i=1}^{n} \sqrt{-\ln \mathrm{y}_{i}} & n
\end{array}\right]\left[\begin{array}{c}
\sum_{i=1}^{n} x_{i} \\
D \sum_{i=1}^{n} x_{i} \sqrt{-\ln \mathrm{y}_{i}}
\end{array}\right],
\end{gathered}
$$

so

$$
\begin{gathered}
\widehat{m}=\frac{\sum_{i=1}^{n} x_{i} \sum_{i=1}^{n}\left(-\ln \mathrm{y}_{i}\right)-\sum_{i=1}^{n} \sqrt{-\ln \mathrm{y}_{i}} \sum_{i=1}^{n} x_{i} \sqrt{-\ln \mathrm{y}_{i}}}{n \sum_{i=1}^{n}\left(-\ln \mathrm{y}_{i}\right)-\left(\sum_{i=1}^{n} \sqrt{-\ln \mathrm{y}_{i}}\right)^{2}} \\
\hat{\sigma}=\frac{-D \sum_{i=1}^{n} x_{i} \sum_{i=1}^{n} \sqrt{-\ln \mathrm{y}_{i}}+D n \sum_{i=1}^{n} x_{i} \sqrt{-\ln \mathrm{y}_{i}}}{n \sum_{i=1}^{n}\left(-\ln \mathrm{y}_{i}\right)-\left(\sum_{i=1}^{n} \sqrt{-\ln \mathrm{y}_{i}}\right)^{2}} .
\end{gathered}
$$

As the number of partition approaches infinity, we have that

$$
\begin{gathered}
\sum_{i=1}^{n}\left(-\ln \mathrm{y}_{i}\right)=\sum_{i=1}^{n}\left(-\ln \frac{i}{n}\right)=n \sum_{i=1}^{n} \frac{1}{n}\left(-\ln \frac{i}{n}\right) \approx n \int_{0}^{1}-\ln x d x=n \\
\sum_{i=1}^{n} \sqrt{-\ln \mathrm{y}_{i}}=n \sum_{i=1}^{n} \frac{1}{n} \sqrt{-\ln \frac{i}{n}} \approx n \int_{0}^{1} \sqrt{-\ln x} d x=n \frac{\sqrt{\pi}}{2}
\end{gathered}
$$




$$
\sum_{i=1}^{n} \frac{i}{n} \sqrt{-\ln y_{i}}=n \sum_{i=1}^{n} \frac{1}{n} \frac{i}{n} \sqrt{-\ln \frac{i}{n}} \approx n \int_{0}^{1} x \sqrt{-\ln x} d x=n \frac{\sqrt{\pi / 2}}{4}
$$

It follows that

$$
\begin{gathered}
\sum_{i=1}^{n} x_{i}=\sum_{i=1}^{n}\left(A+B \frac{i}{n}+C \sqrt{-\ln \frac{i}{n}}\right)=A n+\frac{(n+1) B}{2}+C n \frac{\sqrt{\pi}}{2} \\
\sum_{i=1}^{n} x_{i} \sqrt{-\ln \mathrm{y}_{i}}=\sum_{i=1}^{n}\left(A+B \frac{i}{n}+C \sqrt{-\ln \frac{i}{n}}\right) \sqrt{-\ln \mathrm{y}_{i}}=A n \frac{\sqrt{\pi}}{2}+B \frac{n \sqrt{\pi / 2}}{4}+C n
\end{gathered}
$$

so

$$
\begin{gathered}
\widehat{m}=A+B \frac{8+8 n-\sqrt{2} \pi n}{4 n(4-\pi)} \\
\hat{\sigma}=D C+D B \frac{(-2+(-2+\sqrt{2}) n) \sqrt{\pi}}{2 n(4-\pi)} .
\end{gathered}
$$

By letting $n \rightarrow \infty$, it follows that

$$
\begin{gathered}
\widehat{m}=A+B \frac{8-\sqrt{2} \pi}{4(4-\pi)}=A+1.0359642 B \\
\hat{\sigma}=D C+D B \frac{(-2+\sqrt{2}) \sqrt{\pi}}{2(4-\pi)}=D C-0.604771 D B .
\end{gathered}
$$

We use the normal fuzzy number $C\left(m_{C}, \sigma_{C}\right)$ with $C_{\alpha}=\left[L_{C}, U_{C}\right]$ to approximate the fuzzy number $\gamma A+\beta B$. Firstly, we approximate $U_{\mathrm{C}}$ by the following membership function

$$
\mathrm{y}_{i}=e^{-\left(\frac{x_{i}-m_{U}}{\sigma_{U}}\right)^{2}}
$$

The regression model is

$$
x_{i}=m_{U}+\sigma_{U} \sqrt{-\ln \mathrm{y}_{i}}, i=1,2, \ldots, n .
$$

where $x_{i}=\beta m+\gamma d-\gamma(d-c) \frac{i}{n}+\beta \sigma \sqrt{-\ln \frac{i}{n}}, \mathrm{y}_{i}=i / n, i=1,2, \ldots, n$. Applying Theorem 2 with $A=\beta m+\gamma d, B=-\gamma(d-c), C=\beta \sigma$ and $D=1$, we obtain 


$$
\begin{aligned}
& m_{U}=\beta m+\gamma d-\gamma(d-c) \frac{8+8 n-\sqrt{2} \pi n}{4 n(4-\pi)} \\
& \sigma_{U}=\beta \sigma-\gamma(d-c) \frac{(-2+(-2+\sqrt{2}) n) \sqrt{\pi}}{2 n(4-\pi)}
\end{aligned}
$$

By letting $n \rightarrow \infty$, it follows that

$$
\begin{gathered}
m_{U}=\beta m+\gamma d-\gamma(d-c) \frac{8-\sqrt{2} \pi}{4(4-\pi)}=\beta m+\gamma d-1.0359642 \gamma(d-c) \\
\sigma_{U}=\beta \sigma-\gamma(d-c) \frac{(-2+\sqrt{2}) \sqrt{\pi}}{2(4-\pi)}=\beta \sigma+0.604771 \gamma(d-c) .
\end{gathered}
$$

Secondly, approximate $L_{\mathrm{C}}$ by the following membership function

$$
\mathrm{y}_{i}=e^{-\left(\frac{x_{i}-m_{L}}{\sigma_{L}}\right)^{2}} .
$$

Consider the following regression model

$$
x_{i}=m_{L}-\sigma_{L} \sqrt{-\ln \mathrm{y}_{i}}
$$

where $x_{i}=\beta m+\gamma a+\gamma(b-a) \frac{i}{n}-\beta \sigma \sqrt{-\ln \frac{i}{n}}, \quad y_{i}=i / n, i=1,2, \ldots, n$. A similar argument shows that

$$
\begin{aligned}
& m_{L}=\beta m+\gamma a+\gamma(b-a) \frac{8+8 n-\sqrt{2} \pi n}{4 n(4-\pi)} \\
& \sigma_{L}=\beta \sigma-\gamma(b-a) \frac{(-2+(-2+\sqrt{2}) n) \sqrt{\pi}}{2 n(4-\pi)} .
\end{aligned}
$$

By letting $n \rightarrow \infty$, we have that

$$
\begin{gathered}
m_{L}=\beta m+\gamma a+\gamma(b-a) \frac{8-\sqrt{2} \pi}{4(4-\pi)}=\beta m+\gamma a+1.0359642 \gamma(b-a) \\
\sigma_{L}=\beta \sigma+\frac{(2-\sqrt{2}) \sqrt{\pi} \gamma(b-a)}{2(4-\pi)}=\beta \sigma+0.604771 \gamma(b-a) .
\end{gathered}
$$

To illustrate the addition operation of the mixed fuzzy numbers, we present an example with the trapezoidal fuzzy number $A(4,10,17,26)$ and the normal fuzzy 
number $B(13,5)$. If $n=100, \beta=\gamma=10$, then $C=10 A+10 B$ with $C_{\alpha}=$ $\left[L_{\mathrm{C}}, U_{\mathrm{C}}\right]$ is as follows.

For the $U_{\mathrm{C}}$, we have that

$$
\begin{gathered}
\mathrm{y}_{i}=e^{-\left(\frac{x_{i}-m_{U}}{\sigma_{U}}\right)^{2}} \\
m_{U}=\beta m+\gamma d-\gamma(d-c) \frac{8+8 n-\sqrt{2} \pi n}{4 n(4-\pi)}=294.666 \\
\sigma_{U}=\beta \sigma-\gamma(d-c) \frac{(-2+(-2+\sqrt{2}) n) \sqrt{\pi}}{2 n(4-\pi)}=106.288 .
\end{gathered}
$$

so $U_{\mathrm{C}}$ is approximated by the normal fuzzy number with $(m, \sigma)=$ $(294.666,106.288)$.

Similarly, for the $L_{\mathrm{C}}$, we obtain that

$$
\begin{gathered}
\mathrm{y}_{i}=e^{-\left(\frac{x_{i}-m_{L}}{\sigma_{L}}\right)^{2}} \\
m_{L}=\beta m+\gamma a+\gamma(b-a) \frac{8+8 n-\sqrt{2} \pi n}{4 n(4-\pi)}=232.556 \\
\sigma_{L}=\beta \sigma-\gamma(b-a) \frac{(-2+(-2+\sqrt{2}) n) \sqrt{\pi}}{2 n(4-\pi)}=87.5251
\end{gathered}
$$

so $L_{\mathrm{C}}$ is approximated by the normal fuzzy number with $(m, \sigma)=$ $(232.556,87.5251)$

For purposes of comparison, we measure the value of the relative error (RE), which is the deviation from the area of the exact membership function. Given $\alpha=i / n$, $i=1,2, \ldots, n$, for the $U_{\mathrm{C}}$, the exact and approximated membership function are

$$
x_{i}=\beta m+\gamma \mathrm{d}-\gamma(\mathrm{d}-\mathrm{c}) i / n+\beta \sigma \sqrt{-\ln (i / n)}
$$


and

$$
\widehat{x}_{\imath}=m_{U}+\sigma_{U} \sqrt{-\ln (i / n)}
$$

respectively. The area difference between the exact and approximated membership functions for the $U_{\mathrm{C}}$ is expressed as

$$
\mathrm{AD}_{U}=\sum_{i=1}^{n}\left|\beta m+\gamma \mathrm{d}-\frac{\gamma(\mathrm{d}-\mathrm{c}) i}{n}+\beta \sigma \sqrt{-\ln \left(\frac{i}{n}\right)}-\left(m_{U}+\sigma_{U} \sqrt{-\ln (i / n)}\right)\right| / n .
$$

Similarly, the area difference between the exact and approximated membership functions for the $L_{\mathrm{C}}$ is

$$
\mathrm{AD}_{L}=\sum_{i=1}^{n}\left|\beta m+\gamma \mathrm{a}+\gamma(\mathrm{b}-\mathrm{a}) \frac{i}{n}-\beta \sigma \sqrt{-\ln \left(\frac{i}{n}\right)}-\left(m_{L}-\sigma_{L} \sqrt{-\ln (i / n)}\right)\right| / n .
$$

Therefore, the relative error is

$$
\mathrm{RE}=\frac{\mathrm{AD}_{U}+\mathrm{AD}_{L}}{\sum_{i=1}^{n} \mid \beta m+\gamma \mathrm{d}-\frac{\gamma(\mathrm{d}-\mathrm{c}) i}{n}+\beta \sigma \sqrt{-\ln \left(\frac{i}{n}\right)}-\left(\beta m+\gamma \mathrm{a}+\gamma(\mathrm{b}-\mathrm{a}) \frac{i}{n}-\beta \sigma \sqrt{\left.-\ln \left(\frac{i}{n}\right)\right)} \mid / n\right.} \times 100 \%
$$

For the same example, we analyze the effect of the number of partition on the approximated membership function. Table 1 displays the values of $m_{U}, \sigma_{U}, m_{L}, \sigma_{L}$, $\mathrm{AD}_{U}, \mathrm{AD}_{L}$ and $\mathrm{RE}$ for $n=10,100, \ldots, 1000000$. Of which the best RE is $2.9734 \%$ for $n=100$. The average RE is $3.8265 \%$. Therefore, the best number of partition is $n=100$.

We now consider the subtraction of the mixed fuzzy numbers $A(a, b, c, d)$ and $B(m, \sigma)$ with

$$
A_{\alpha}=\left[L_{1}, U_{1}\right]=[a+(b-a) \alpha, d-(d-c) \alpha]
$$




$$
B_{\alpha}=\left[L_{2}, U_{2}\right]=[m-\sigma \sqrt{-\ln \alpha}, m+\sigma \sqrt{-\ln \alpha}] .
$$

Let $\gamma, \beta \geq 0$, the subtraction operation $-\gamma A-\beta B$ is

$$
\begin{gathered}
C_{\alpha}=\left[L_{\mathrm{C}}, U_{\mathrm{C}}\right]=-\gamma A_{\alpha}-\beta B_{\alpha}=\left[-\gamma U_{1}-\beta U_{2},-\gamma L_{1}-\beta L_{2}\right] \\
=[-\beta m-\gamma \mathrm{d}+\gamma(\mathrm{d}-\mathrm{c}) \alpha-\beta \sigma \sqrt{-\ln \alpha},-\beta m-\gamma \mathrm{a}-\gamma(\mathrm{b}-\mathrm{a}) \alpha+\beta \sigma \sqrt{-\ln \alpha}] .
\end{gathered}
$$

We use the normal fuzzy number $C\left(m_{C}, \sigma_{C}\right)$ to approximate the fuzzy number $-\gamma A-\beta B$. Firstly, we consider $U_{\mathrm{C}}$ with membership function

$$
\mathrm{y}_{i}=e^{-\left(\frac{x_{i}-m_{U}}{\sigma_{U}}\right)^{2}}
$$

The regression model is

$$
x_{i}=m_{U}+\sigma_{U} \sqrt{-\ln \mathrm{y}_{i}} .
$$

where $x_{i}=-\beta m-\gamma a-\gamma(b-a) \frac{i}{n}+\beta \sigma \sqrt{-\ln \frac{i}{n}}, \mathrm{y}_{i}=i / n, i=1,2, \ldots, n$.

By Theorem 2, a similar argument shows that

$$
\begin{gathered}
m_{U}=-\beta m-\gamma a-\gamma(b-a) \frac{8+8 n-\sqrt{2} \pi n}{4 n(4-\pi)} \\
\sigma_{U}=\beta \sigma-\gamma(b-a) \frac{(-2+(-2+\sqrt{2}) n) \sqrt{\pi}}{2 n(4-\pi)} .
\end{gathered}
$$

By letting $n \rightarrow \infty$, we have that

$$
\begin{gathered}
m_{U}=-\beta m-\gamma a-\gamma(b-a) \frac{8-\sqrt{2} \pi}{4(4-\pi)}=-\beta m-\gamma a-1.0359642 \gamma(b-a) \\
\sigma_{U}=\beta \sigma+\frac{(2-\sqrt{2}) \sqrt{\pi} \gamma(b-a)}{2(4-\pi)}=\beta \sigma+0.604771 \gamma(b-a) .
\end{gathered}
$$

Secondly, for the $L_{\mathrm{C}}$, we have that

$$
m_{L}=-\beta m-\gamma d+\gamma(d-c) \frac{8+8 n-\sqrt{2} \pi n}{4 n(4-\pi)}
$$




$$
\sigma_{L}=\beta \sigma-\gamma(d-c) \frac{(-2+(-2+\sqrt{2}) n) \sqrt{\pi}}{2 n(4-\pi)}
$$

By letting $n \rightarrow \infty$, it follows that

$$
\begin{gathered}
m_{L}=-\beta m-\gamma d+\gamma(d-c) \frac{8-\sqrt{2} \pi}{4(4-\pi)}=-\beta m-\gamma d+1.0359642 \gamma(d-c) \\
\sigma_{L}=\beta \sigma-\gamma(d-c) \frac{(-2+\sqrt{2}) \sqrt{\pi}}{2(4-\pi)}=\beta \sigma+0.604771 \gamma(d-c) .
\end{gathered}
$$

Theorem 3. Consider the trapezoidal fuzzy number $A(a, b, c, d)$ and the normal fuzzy number $B(m, \sigma)$. Let $\gamma, \beta \geq 0$. Then

(1) The addition operation $\gamma A+\beta B$ is approximated by the normal fuzzy number $C\left(m_{C}, \sigma_{C}\right)$ with $C_{\alpha}=\left[L_{C}, U_{C}\right]$. The approximated membership function of $U_{\mathrm{C}}$ is

$$
\mathrm{y}_{i}=e^{-\left(\frac{x_{i}-m_{U}}{\sigma_{U}}\right)^{2}}
$$

where

$$
\begin{gathered}
m_{U}=\beta m+\gamma d-\gamma(d-c) \frac{8+8 n-\sqrt{2} \pi n}{4 n(4-\pi)} \approx \beta m+\gamma d-1.0359642 \gamma(d-c) \\
\sigma_{U}=\beta \sigma-\gamma(d-c) \frac{(-2+(-2+\sqrt{2}) n) \sqrt{\pi}}{2 n(4-\pi)} \approx \beta \sigma+0.604771 \gamma(d-c)
\end{gathered}
$$

The approximated membership function of $L_{\mathrm{C}}$ is

$$
\mathrm{y}_{i}=e^{-\left(\frac{x_{i}-m_{L}}{\sigma_{L}}\right)^{2}}
$$

where

$$
\begin{gathered}
m_{L}=\beta m+\gamma a+\gamma(b-a) \frac{8+8 n-\sqrt{2} \pi n}{4 n(4-\pi)} \approx \beta m+\gamma a+1.0359642 \gamma(b-a) \\
\sigma_{L}=\beta \sigma-\gamma(b-a) \frac{(-2+(-2+\sqrt{2}) n) \sqrt{\pi}}{2 n(4-\pi)} \approx \beta \sigma+0.604771 \gamma(b-a) .
\end{gathered}
$$

(2) The subtraction operation $-\gamma A-\beta B$ is approximated by the normal fuzzy number 
$C\left(m_{C}, \sigma_{C}\right)$ with $C_{\alpha}=\left[L_{C}, U_{C}\right]$. The approximated membership function of $U_{\mathrm{C}}$ is

$$
\mathrm{y}_{i}=e^{-\left(\frac{x_{i}-m_{U}}{\sigma_{U}}\right)^{2}}
$$

where

$$
\begin{gathered}
m_{U}=-\beta m-\gamma a-\gamma(b-a) \frac{8+8 n-\sqrt{2} \pi n}{4 n(4-\pi)} \approx-\beta m-\gamma a-1.0359642 \gamma(b-a) \\
\sigma_{U}=\beta \sigma-\gamma(b-a) \frac{(-2+(-2+\sqrt{2}) n) \sqrt{\pi}}{2 n(4-\pi)} \approx \beta \sigma+0.604771 \gamma(b-a) .
\end{gathered}
$$

The approximated membership function of $L_{\mathrm{C}}$ is

$$
\mathrm{y}_{i}=e^{-\left(\frac{x_{i}-m_{L}}{\sigma_{L}}\right)^{2}}
$$

where

$$
\begin{gathered}
m_{L}=-\beta m-\gamma d+\gamma(d-c) \frac{8+8 n-\sqrt{2} \pi n}{4 n(4-\pi)} \approx-\beta m-\gamma d+1.0359642 \gamma(d-c) \\
\sigma_{L}=\beta \sigma-\gamma(d-c) \frac{(-2+(-2+\sqrt{2}) n) \sqrt{\pi}}{2 n(4-\pi)} \approx \beta \sigma+0.604771 \gamma(d-c) .
\end{gathered}
$$

To illustrate the subtraction of mixed fuzzy numbers, the same example with $A(4,10,17,26), B(13,5), n=100, \beta=\gamma=10$, and $C=-10 A-10 B$ is as follows.

For the $U_{\mathrm{C}}$, we have that

$$
\begin{gathered}
m_{U}=-\beta m-\frac{(8+(8-\sqrt{2} \pi) n) \gamma b+(-8+(8+(-4+\sqrt{2}) \pi) n) \gamma a}{4 n(4-\pi)}=-232.556 \\
\sigma_{U}=\beta \sigma+\frac{(2+(2-\sqrt{2}) n) \sqrt{\pi} \gamma(\mathrm{b}-\mathrm{a})}{2 n(4-\pi)}=87.5251
\end{gathered}
$$

so $U_{\mathrm{C}}$ is approximated by the normal fuzzy number with $(m, \sigma)=$ $(-232.996,87.4381)$ 
Similarly, for the $L_{\mathrm{C}}$, we obtain that

$$
\begin{gathered}
m_{L}=-\beta m-\frac{(8+(8-\sqrt{2} \pi) n) \gamma c+(-8+(8+(-4+\sqrt{2}) \pi) n) \gamma d}{4 n(4-\pi)}=-294.666 \\
\sigma_{L}=\beta \sigma+\frac{(2+(2-\sqrt{2}) n) \sqrt{\pi} \gamma(\mathrm{d}-\mathrm{c})}{2 n(4-\pi)}=106.288,
\end{gathered}
$$

so $L_{\mathrm{C}}$ is approximated by the normal fuzzy number with $(m, \sigma)=$ $(-294.666,106.288)$

\section{Fuzzy Sample Mean}

An application of the addition and subtraction operations of the same and mixed fuzzy numbers to the fuzzy sample mean is proposed in this section. Let $\mathrm{X}_{1}\left(a_{1}, b_{1}, c_{1}, d_{1}\right), \mathrm{X}_{2}\left(a_{2}, b_{2}, c_{2}, d_{2}\right), \ldots, \mathrm{X}_{n}\left(a_{n}, b_{n}, c_{n}, d_{n}\right)$ be a fuzzy sample. We have that

$$
\mathrm{X}_{i \alpha}=\left[L_{i \alpha}, U_{i \alpha}\right]=\left[a_{i}+\left(b_{i}-a_{i}\right) \alpha, d_{i}-\left(d_{i}-c_{i}\right) \alpha\right], i=1,2, \ldots, n
$$

It follows that the fuzzy sample mean $\overline{\mathrm{X}}$ is

$$
\overline{\mathrm{X}}_{\alpha}=\frac{1}{n} \sum_{i=1}^{n} X_{i \alpha}=\frac{1}{n} \sum_{i=1}^{n}\left[a_{i}+\left(b_{i}-a_{i}\right) \alpha, d_{i}-\left(d_{i}-c_{i}\right) \alpha\right]
$$

so the fuzzy sample mean is $\overline{\mathrm{X}}(\bar{a}, \bar{b}, \bar{c}, \bar{d})$ with membership function

$$
\mu_{\overline{\mathrm{X}}}(x)= \begin{cases}\frac{x-\bar{a}}{\bar{b}-\bar{a}}, & \bar{a} \leq x \leq \bar{b} \\ 1, & \bar{b} \leq x \leq \bar{c} \\ \overline{\bar{d}-x} & \bar{c} \leq x \leq \bar{d}\end{cases}
$$

where $\bar{a}=\frac{1}{n} \sum_{i=1}^{n} a_{i}, \bar{b}=\frac{1}{n} \sum_{i=1}^{n} b_{i}, \bar{c}=\frac{1}{n} \sum_{i=1}^{n} c_{i}$ and $\bar{d}=\frac{1}{n} \sum_{i=1}^{n} d_{i}$. 
For the normal fuzzy numbers, let $\mathrm{X}_{1}\left(m_{1}, \sigma_{1}\right), \mathrm{X}_{2}\left(m_{2}, \sigma_{2}\right), \ldots, \mathrm{X}_{n}\left(m_{n}, \sigma_{n}\right)$ be a fuzzy sample. From

$$
\mathrm{X}_{i \alpha}=\left[L_{i \alpha}, U_{i \alpha}\right]=\left[m_{i}-\sigma_{i} \sqrt{-\ln \alpha}, m_{i}+\sigma_{i} \sqrt{-\ln \alpha}\right] \cdot i=1,2, \ldots, n,
$$

and

$$
\overline{\mathrm{X}}_{\alpha}=\frac{1}{n} \sum_{i=1}^{n} X_{i \alpha}=\frac{1}{n} \sum_{i=1}^{n}\left[m_{i}-\sigma_{i} \sqrt{-\ln \alpha}, m_{i}+\sigma_{i} \sqrt{-\ln \alpha}\right]
$$

it follows that the membership function of fuzzy sample mean $\overline{\mathrm{X}}(\bar{m}, \bar{\sigma})$ is

$$
\mu_{\overline{\mathrm{X}}}(x)=e^{-\left(\frac{x-\bar{m}}{\bar{\sigma}}\right)^{2}}
$$

where $\bar{m}=\frac{1}{n} \sum_{i=1}^{n} m_{i}$ and $\bar{\sigma}=\frac{1}{n} \sum_{i=1}^{n} \sigma_{i}$.

We now consider the mixed fuzzy numbers. Let $\mathrm{X}_{1}\left(a_{1}, b_{1}, c_{1}, d_{1}\right)$, $\mathrm{X}_{2}\left(a_{2}, b_{2}, c_{2}, d_{2}\right), \ldots, \quad \mathrm{X}_{n_{1}}\left(a_{n}, b_{n}, c_{n}, d_{n}\right), \mathrm{X}_{n_{1}+1}\left(m_{1}, \sigma_{1}\right), \mathrm{X}_{n_{1}+2}\left(m_{2}, \sigma_{2}\right), \ldots$ $\mathrm{X}_{n_{1}+n_{2}}\left(m_{n}, \sigma_{n}\right)$ be a fuzzy sample. We obtain that

$$
\begin{gathered}
\mathrm{X}_{i \alpha}=\left[L_{i \alpha}, U_{i \alpha}\right]=\left[a_{i}+\left(b_{i}-a_{i}\right) \alpha, d_{i}-\left(d_{i}-c_{i}\right) \alpha\right], i=1,2, \ldots, n_{1} \\
\mathrm{X}_{i \alpha}=\left[L_{i \alpha}, U_{i \alpha}\right]=\left[m_{i-n_{1}}-\sigma_{i-n_{1}} \sqrt{-\ln \alpha}, m_{i-n_{1}}+\sigma_{i-n_{1}} \sqrt{-\ln \alpha}\right], i=n_{1}+1, n_{1}+ \\
2, \ldots, n_{1}+n_{2} .
\end{gathered}
$$

The fuzzy sample mean $\overline{\mathrm{X}}$ is

$$
\begin{gathered}
\overline{\mathrm{X}}_{\alpha}=\left[\bar{X}_{\alpha}^{L}, \bar{X}_{\alpha}^{U}\right]=\frac{1}{n_{1}+n_{2}} \sum_{i=1}^{n_{1}+n_{2}} X_{i \alpha}=\frac{1}{n_{1}+n_{2}}\left(\sum_{i=1}^{n_{1}}\left[a_{i}+\left(b_{i}-a_{i}\right) \alpha, d_{i}-\left(d_{i}-c_{i}\right) \alpha\right]+\right. \\
\left.\sum_{i=1}^{n_{2}}\left[m_{i}-\sigma_{i} \sqrt{-\ln \alpha}, m_{i}+\sigma_{i} \sqrt{-\ln \alpha}\right]\right)
\end{gathered}
$$

so 


$$
\bar{X}_{\alpha}^{L}=\frac{n_{1}}{n_{1}+n_{2}} \bar{a}+\frac{n_{1}}{n_{1}+n_{2}}(\bar{b}-\bar{a}) \alpha+\frac{n_{2}}{n_{1}+n_{2}} \bar{m}-\frac{n_{2}}{n_{1}+n_{2}} \bar{\sigma} \sqrt{-\ln \alpha}
$$

and

$$
\bar{X}_{\alpha}^{U}=\frac{n_{1}}{n_{1}+n_{2}} \bar{d}-\frac{n_{1}}{n_{1}+n_{2}}(\bar{d}-\bar{c}) \alpha+\frac{n_{2}}{n_{1}+n_{2}} \bar{m}+\frac{n_{2}}{n_{1}+n_{2}} \bar{\sigma} \sqrt{-\ln \alpha}
$$

where $\bar{a}=\frac{1}{n_{1}} \sum_{i=1}^{n_{1}} a_{i}, \bar{b}=\frac{1}{n_{1}} \sum_{i=1}^{n_{1}} b_{i}, \bar{c}=\frac{1}{n_{1}} \sum_{i=1}^{n_{1}} c_{i}, \bar{d}=\frac{1}{n_{1}} \sum_{i=1}^{n_{1}} d_{i}, \bar{m}=\frac{1}{n_{2}} \sum_{i=1}^{n_{2}} m_{i}$ and $\bar{\sigma}=\frac{1}{n_{2}} \sum_{i=1}^{n_{2}} \sigma_{i}$

We use the normal fuzzy number $\overline{\mathrm{X}}\left(m_{\overline{\mathrm{X}}}, \sigma_{\overline{\mathrm{X}}}\right)$ to approximate $\overline{\mathrm{X}}$. For the $U_{\overline{\mathrm{X}}}$, a similar argument shows that the regression model is

$$
x_{i}=m_{U}+\sigma_{U} \sqrt{-\ln \mathrm{y}_{i}}
$$

where $x_{i}=\frac{n_{1} \bar{d}+n_{2} \bar{m}}{n_{1}+n_{2}}-\frac{n_{1}(\bar{d}-\bar{c})}{n_{1}+n_{2}} \frac{i}{n}+\frac{n_{2} \bar{\sigma}}{n_{1}+n_{2}} \sqrt{-\ln \frac{i}{n}}, \mathrm{y}_{i}=\frac{i}{n}, i=1,2, \ldots, n_{1}+n_{2}$.

From Theorem 2, the least squares estimators are

$$
\begin{gathered}
\widehat{m}=\frac{n_{1} \bar{d}+n_{2} \bar{m}}{n_{1}+n_{2}}-\frac{n_{1}(\bar{d}-\bar{c})}{n_{1}+n_{2}} \frac{8+8 n+\sqrt{2} \pi n}{4 n(4-\pi)} \\
\hat{\sigma}=\frac{n_{2} \bar{\sigma}}{n_{1}+n_{2}}-\frac{n_{1}(\bar{d}-\bar{c})}{n_{1}+n_{2}} \frac{(-2+(-2+\sqrt{2}) n) \sqrt{\pi}}{2 n(4-\pi)} .
\end{gathered}
$$

As $n \rightarrow \infty$, it follows that

$$
\begin{gathered}
\widehat{m}=\frac{n_{1} \bar{d}+n_{2} \bar{m}}{n_{1}+n_{2}}-\frac{n_{1}(\bar{d}-\bar{c})}{n_{1}+n_{2}} \frac{8+\sqrt{2} \pi}{4(4-\pi)}=\frac{n_{1} \bar{d}+n_{2} \bar{m}}{n_{1}+n_{2}}-1.0359642 \frac{8+\sqrt{2} \pi}{4(4-\pi)} \\
\hat{\sigma}=\frac{n_{2} \bar{\sigma}}{n_{1}+n_{2}}-\frac{n_{1}(\bar{d}-\bar{c})}{n_{1}+n_{2}} \frac{(-2+\sqrt{2}) \sqrt{\pi}}{2(4-\pi)}=\frac{n_{2} \bar{\sigma}}{n_{1}+n_{2}}+0.604771 \frac{n_{1}(\bar{d}-\bar{c})}{n_{1}+n_{2}} .
\end{gathered}
$$

Similarly, for the $L_{\mathrm{C}}$, we have that the regression model is

$$
x_{i}=m_{L}-\sigma_{L} \sqrt{-\ln \mathrm{y}_{i}}
$$

where $x_{i}=\frac{n_{1} \bar{a}+n_{2} \bar{m}}{n_{1}+n_{2}}+\frac{n_{1}}{n_{1}+n_{2}}(\bar{b}-\bar{a}) \frac{i}{n}-\frac{n_{2}}{n_{1}+n_{2}} \bar{\sigma} \sqrt{-\ln \frac{i}{n}}, \mathrm{y}_{i}=\frac{i}{n}, i=1,2, \ldots, n_{1}+n_{2}$. 
Then the least squares estimators are

$$
\begin{gathered}
\hat{m}=\frac{n_{1} \bar{a}+n_{2} \bar{m}}{n_{1}+n_{2}}+\frac{n_{1}}{n_{1}+n_{2}}(\bar{b}-\bar{a}) \frac{8+8 n+\sqrt{2} \pi n}{4 n(4-\pi)} \\
\hat{\sigma}=\frac{n_{2}}{n_{1}+n_{2}} \bar{\sigma}-\frac{n_{1}}{n_{1}+n_{2}}(\bar{b}-\bar{a}) \frac{(-2+(-2+\sqrt{2}) n) \sqrt{\pi}}{2 n(4-\pi)} .
\end{gathered}
$$

As $n \rightarrow \infty$, it follows that

$$
\begin{aligned}
& \widehat{m}=\frac{n_{1} \bar{a}+n_{2} \bar{m}}{n_{1}+n_{2}}+\frac{n_{1}}{n_{1}+n_{2}}(\bar{b}-\bar{a}) \frac{8+\sqrt{2} \pi}{4(4-\pi)}=\frac{n_{1} \bar{a}+n_{2} \bar{m}}{n_{1}+n_{2}}+1.0359642 \frac{n_{1}}{n_{1}+n_{2}}(\bar{b}-\bar{a}) \\
& \hat{\sigma}=\frac{n_{2}}{n_{1}+n_{2}} \bar{\sigma}-\frac{n_{1}}{n_{1}+n_{2}}(\bar{b}-\bar{a}) \frac{(-2+\sqrt{2}) \sqrt{\pi}}{2(4-\pi)}=\frac{n_{2}}{n_{1}+n_{2}} \bar{\sigma}+0.604771 \frac{n_{1}}{n_{1}+n_{2}}(\bar{b}-\bar{a}) .
\end{aligned}
$$

Theorem 4. (1) Let $\mathrm{X}_{1}\left(a_{1}, b_{1}, c_{1}, d_{1}\right), \mathrm{X}_{2}\left(a_{2}, b_{2}, c_{2}, d_{2}\right), \ldots, \mathrm{X}_{n}\left(a_{n}, b_{n}, c_{n}, d_{n}\right)$ be a fuzzy sample. Then the fuzzy sample mean is $\overline{\mathrm{X}}(\bar{a}, \bar{b}, \bar{c}, \bar{d})$ where $\bar{a}=\frac{1}{n} \sum_{i=1}^{n} a_{i}, \bar{b}=$ $\frac{1}{n} \sum_{i=1}^{n} b_{i}, \bar{c}=\frac{1}{n} \sum_{i=1}^{n} c_{i}$ and $\bar{d}=\frac{1}{n} \sum_{i=1}^{n} d_{i}$

(2) For the normal fuzzy numbers, let $\mathrm{X}_{1}\left(m_{1}, \sigma_{1}\right), \mathrm{X}_{2}\left(m_{2}, \sigma_{2}\right), \ldots, \mathrm{X}_{n}\left(m_{n}, \sigma_{n}\right)$ be a fuzzy sample. Then the fuzzy sample mean is $\overline{\mathrm{X}}(\bar{m}, \bar{\sigma})$ where $\bar{m}=\frac{1}{n} \sum_{i=1}^{n} m_{i}$ and $\bar{\sigma}=\frac{1}{n} \sum_{i=1}^{n} \sigma_{i}$

(3) Let $\mathrm{X}_{1}\left(a_{1}, b_{1}, c_{1}, d_{1}\right), \mathrm{X}_{2}\left(a_{2}, b_{2}, c_{2}, d_{2}\right), \ldots, \mathrm{X}_{n_{1}}\left(a_{n}, b_{n}, c_{n}, d_{n}\right), \mathrm{X}_{n_{1}+1}\left(m_{1}, \sigma_{1}\right)$, $\mathrm{X}_{n_{1}+2}\left(m_{2}, \sigma_{2}\right), \ldots, \mathrm{X}_{n_{1}+n_{2}}\left(m_{n}, \sigma_{n}\right)$ be a fuzzy sample. Then the fuzzy sample mean $\overline{\mathrm{X}}$ is approximated by the normal fuzzy number $\overline{\mathrm{X}}\left(m_{\overline{\mathrm{X}}}, \sigma_{\overline{\mathrm{X}}}\right)$ with $\overline{\mathrm{X}}_{\alpha}=\left[L_{\overline{\mathrm{X}}}, U_{\overline{\mathrm{X}}}\right]$. The approximated membership function of $U_{\overline{\mathrm{X}}}$ is

$$
\mathrm{y}_{i}=e^{-\left(\frac{x_{i}-m_{U}}{\sigma_{U}}\right)^{2}}
$$

where 


$$
\begin{aligned}
& \widehat{m_{U}}=\frac{n_{1} \bar{d}+n_{2} \bar{m}}{n_{1}+n_{2}}-\frac{n_{1}(\bar{d}-\bar{c})}{n_{1}+n_{2}} \frac{8+8 n+\sqrt{2} \pi n}{4 n(4-\pi)} \approx \frac{n_{1} \bar{d}+n_{2} \bar{m}}{n_{1}+n_{2}}-1.0359642 \frac{8+\sqrt{2} \pi}{4(4-\pi)} \\
& \widehat{\sigma_{U}}=\frac{n_{2} \bar{\sigma}}{n_{1}+n_{2}}-\frac{n_{1}(\bar{d}-\bar{c})}{n_{1}+n_{2}} \frac{(-2+(-2+\sqrt{2}) n) \sqrt{\pi}}{2 n(4-\pi)} \approx \frac{n_{2} \bar{\sigma}}{n_{1}+n_{2}}+0.604771 \frac{n_{1}(\bar{d}-\bar{c})}{n_{1}+n_{2}} .
\end{aligned}
$$

The approximated membership function of $L_{\overline{\mathrm{X}}}$ is

$$
\mathrm{y}_{i}=e^{-\left(\frac{x_{i}-m_{L}}{\sigma_{L}}\right)^{2}}
$$

where

$$
\begin{gathered}
\widehat{m_{L}}=\frac{n_{1} \bar{a}+n_{2} \bar{m}}{n_{1}+n_{2}}+\frac{n_{1}}{n_{1}+n_{2}}(\bar{b}-\bar{a}) \frac{8+8 n+\sqrt{2} \pi n}{4 n(4-\pi)} \approx \frac{n_{1} \bar{a}+n_{2} \bar{m}}{n_{1}+n_{2}}+1.0359642 \frac{n_{1}}{n_{1}+n_{2}}(\bar{b}-\bar{a}) \\
\widehat{\sigma_{L}}=\frac{n_{2}}{n_{1}+n_{2}} \bar{\sigma}-\frac{n_{1}}{n_{1}+n_{2}}(\bar{b}-\bar{a}) \frac{(-2+(-2+\sqrt{2}) n) \sqrt{\pi}}{2 n(4-\pi)} \approx \frac{n_{2}}{n_{1}+n_{2}} \bar{\sigma}+0.604771 \frac{n_{1}}{n_{1}+n_{2}}(\bar{b}-\bar{a}) .
\end{gathered}
$$

\section{Conclusion}

Addition and subtraction operations are two fundamental problems of fuzzy group decision making problems and fuzzy shortest path problems. For the same type of fuzzy numbers, the results of addition and subtraction operations are the same ones. For the mixed fuzzy numbers, Theorem 2 presents the least squares estimators of the normal fuzzy number approximations. By applying Theorem 2, the normal approximations of the addition and subtraction operations are displayed in Theorem 3. An application of Theorem 2 to fuzzy sample mean is shown in Theorem 4. The behaviors of approximated normal fuzzy numbers are the same as those of the normal distributions from the viewpoint of probability. These results provide insights into the normal approximations of mixed fuzzy numbers. The results indicate that the normal 
approximation is an appropriate one to approximate the arithmetic of mixed fuzzy numbers.

Worthy of future research is extending the normal approximations to fuzzy multiplication. In particular, the problem of normal approximations for multiple judges, multiple criteria decision making problem is a subject of considerable ongoing research.

\section{References}

1. Lee, H.S. On fuzzy preference relation in group decision making. Int. J. Comput. Math. 2005, 82, 133-140.

2. Tang, H.C. Decomposition and intersection of two fuzzy numbers for the fuzzy preference relations. Sym. 2017, 9, 228; doi:10.3390/sym9100228.

3. Wang, X.; Kerre, E.E. Reasonable properties for the ordering of fuzzy quantities (I). Fuzzy Sets Syst.2001, 118, 375-385.

4. Wang, X.; Kerre, E.E. Reasonable properties for the ordering of fuzzy quantities (II). Fuzzy Sets Syst.2001, 118, 387-405.

5. Deng, Y.; Chen, Y.; Zhang, Y.; Mahadevan S. Fuzzy Dijkstra algorithm for shortest path problem under uncertain environment. Appl. Soft Comput. 2012, 12, 1231-1237.

6. Niroomand, S.; Mahmoodirad, A.; Heydari, A.; Kardani, F.; Hadi-Vencheh A. An 
extension principle based solution approach for shortest path problem with fuzzy arc lengths. Oper. Res. 2017, 17, 395-411.

7. Tajdin, A; Mahdavi, A; Mahdavi-Amiri, N; Sadeghpour-Gildeh, B. Computing a fuzzy shortest path in a network with mixed fuzzy arc lengths using $\alpha$-cuts. Comput. Math. Appl. 2010, 60, 989-1002.

8. Grzegorzewski, P; Mrówka, E. Trapezoidal approximations of fuzzy numbers. Fuzzy. Sets. Syst. 2005, 153, 115-135.

9. Grzegorzewski, P; Mrówka E Trapezoidal approximations of fuzzy numbers-revisited. Fuzzy. Sets. Syst. 2007, 158, 757-768.

10. Grzegorzewski, P. Trapezoidal approximations of fuzzy numbers preserving the expected interval - algorithms and properties. Fuzzy. Sets. Syst. 2008, 159, 13541364.

11. Yeh, CT. Note on symmetric triangular approximations of fuzzy numbers under a general condition and properties. Soft. Comput. 2017, https://doi.org/10.1007/s00500-017-2692-6. 
Table 1. The area $Q_{i}$ of $i$-th region for eight cases.

\begin{tabular}{|l|l|l|l|l|l|l|l|}
\hline$n$ & \multicolumn{1}{|c|}{$m_{U}$} & \multicolumn{1}{c|}{$\sigma_{U}$} & \multicolumn{1}{c|}{$m_{L}$} & \multicolumn{1}{c|}{$\sigma_{L}$} & \multicolumn{1}{c|}{$\mathrm{AD}_{U}$} & \multicolumn{1}{|c|}{$\mathrm{AD}_{L}$} & $\mathrm{RE}$ \\
\hline 10 & 275.794 & 123.013 & 246.137 & 98.6751 & 9.0043 & 6.00287 & $6.9697 \%$ \\
\hline 100 & 294.666 & 106.288 & 233.556 & 87.5251 & 4.1315 & 2.75434 & $2.9734 \%$ \\
\hline 1000 & 296.554 & 104.615 & 232.298 & 86.4101 & 4.51635 & 3.0109 & $3.2251 \%$ \\
\hline 10000 & 296.742 & 104.448 & 232.172 & 86.2986 & 4.57003 & 3.04668 & $3.2606 \%$ \\
\hline 100000 & 296.761 & 104.431 & 232.159 & 86.2875 & 4.57636 & 3.0509 & $3.2648 \%$ \\
\hline 1000000 & 296.763 & 104.43 & 232.158 & 86.2864 & 4.57707 & 3.05138 & $3.2653 \%$ \\
\hline
\end{tabular}

